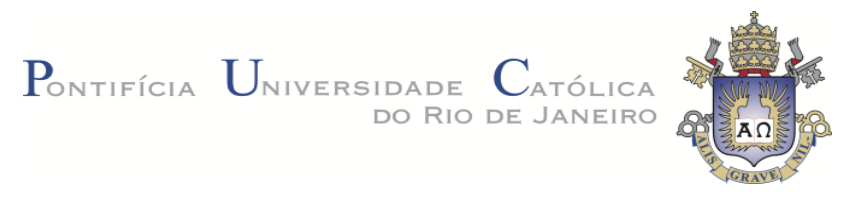

Maria Isabel Ramos Navarro

Síntese e caracterização de ligas Cobre-Níquel contendo nanoparticulas de Alumina

Dissertação apresentada como requisito parcial para obtenção do grau de Mestre pelo Programa de Pós-graduação em Engenharia de Materiais e de Processos Químicos e Metalúrgicos do Departamento de Engenharia de Materiais do Centro Técnico Científico da PUC-Rio.

Orientador: Prof. Eduardo de Albuquerque Brocchi Co-Orientador: Rogério Navarro Correia de Siqueira 
Maria Isabel Ramos Navarro

\section{Síntese e caracterização de ligas Cobre-Níquel contendo nanoparticulas de Alumina}

Dissertação apresentada como requisito parcial para obtenção do grau de Mestre pelo Programa de Pós-graduação em Engenharia de Materiais e de Processos Químicos e Metalúrgicos do Departamento de Engenharia de Materiais do Centro Técnico Científico da PUC-Rio. Aprovada pela Comissão Examinadora abaixo assinada.

Prof. Eduardo de Albuquerque Brocchi Orientador e Presidente Departamento de Engenharia de Materiais - PUC Rio

Prof. Rogério Navarro Correia de Siqueira Co-Orientador Departamento de Engenharia de Materiais - PUC Rio

Prof. Eduardo de Sousa Lima Instituto Militar de Engenharia - IME

Prof. Francisco José Moura Departamento de Engenharia de Materiais - PUC Rio

Prof $^{\circ}$. José Eugenio Leal Coordenador Setorial de Pós-Graduação do Centro Técnico Científico da PUC- Rio 
Todos os direitos reservados. É proibida a reprodução total ou parcial do trabalho sem autorização da universidade, do autor e do orientador.

\section{Maria Isabel Ramos Navarro}

Graduou-se em Engenharia Química na Universidade Nacional de Colômbia em 2010.

Ficha Catalográfica

Navarro, Maria Isabel Ramos
Síntese e caracterização de ligas Cobre-Níquel contendo
nanoparticulas de Alumina / Maria Isabel Ramos Navarro;
orientador: Eduardo de Albuquerque Brocchi; co-orientador:
Rogério Navarro Correia de Siqueira. - 2013 .
\[ 122 \text { f. : il. (color.) ; } 30 \mathrm{~cm} \]
Dissertação (mestrado)-Pontifícia Universidade Católica do Rio de Janeiro, Departamento de Engenharia de Materiais, 2013.

Inclui bibliografia

1. Engenharia de materiais - Teses. 2. Compósitos $\mathrm{CuNi} / \mathrm{Al}_{2} \mathrm{O}_{3}$. 3. Decomposição térmica de nitratos. 4. Redução seletiva com $\mathrm{H}_{2}$. I. Brocchi, Eduardo de Albuquerque. II. Siqueira, Rogerio Navarro Correia de. III. Pontifícia Universidade Católica do Rio de Janeiro. Departamento de Engenharia de Materiais. IV. Título.

CDD: 620.11 
A las esperanzas del pasado que permitieron este futuro mientras muchas cosas se desvanecían.

A Teresita y Héctor 


\section{Agradecimentos}

A conclusão do mestrado reflete uma importante etapa de construção pessoal e profissional. Tornando-se impossível descrever neste curto espaço toda a gratidão que sinto pelas múltiplas pessoas e entidades que contribuíram no decorrer da pesquisa, por isto, para cada um de vocês, meu mais sincero, carinhoso e breve agradecimento:

Ao meu caríssimo orientador Dr. Eduardo de Albuquerque Brocchi ( $D u d u)$, pela confiança depositada, pelo trato paciente e carinhoso, por permitir-me trabalhar em seu grupo de pesquisa, e principalmente por me mostrar tantos novos caminhos.

Ao co-orientador e grande pesquisador Dr. Rogério Navarro. Poucos têm a sorte de trabalhar com profissionais tão competentes quanto você. Rogério com brilhantismo, paciência e empenho me ajudou e incentivou em todas as etapas do trabalho. Sem seu empenho não teria sido possível! "Muito obrigada, Rogério".

À pupila e nova grande amiga Dra. Natasha Midori Suguihiro, por sua parceria incansável e constante, assim como também por ter compartilhado com grande entusiasmo e paciência seus conhecimentos de microscopia. Nata, obrigada por doar-me seu valioso tempo, dedicado a revisões e discussões para que eu conseguisse sempre ter claro o caminho escolhido.

Agradeço ao companheiro e grande amigo Rodrigo F. M. de Souza, pelos ensinamentos, pelo carinho e principalmente pela paciência, dedicação e bom humor nos momentos difíceis.

Ao Dr. Guillermo Solórzano por amavelmente convidar-me a conhecer o mundo da microscopia e por sua ajuda na compreensão das imagens, assim como também a seu grupo de pesquisadores que calorosamente me acolheu e me ajudou, principalmente a Liying Liu Xing, Cilene Labre, Julio Spadotto e 
Natasha Midori.

Aos funcionários do laboratório de Raios X do CBPF, Valeria e Vitor, pela ajuda nas análises de Raios X. Ao Rodrigo Gonçalves, pela caracterização química dos óxidos. Ao Adrian Giassone do ITUC, pelas medidas de microdureza. Ao Rogério Navarro, pela caracterização no MEV de inúmeras amostras. Ao Victor Hugo e ao Carlos Augusto pelo suporte técnico.

Ao Laboratório Multiusuário de Nanotecnologia (LabNano) do Centro Brasileiro de Pesquisas Físicas/Ministério da Ciência, Tecnologia e Inovação (CBPF/MCTI) pelo acesso ao Microscópio Eletrônico de Transmissão.

Aos funcionários e professores do DEMA, especialmente ao Professor Francisco Moura e a Carmem Façanha, por toda atenção dada.

À minhas boas amigas e companheiras, especialmente a Ingrid Milena Reyes, Leslie Lopez e Mariella Cortez, agradeço por um dia terem cruzado e compartilhado meu caminho.

À Sra. Gloria e Sr. Alfred, por abrir tão carinhosamente as portas de sua casa e me fazer sentir parte da sua família.

Aos meus anjos, Teresita e Héctor, e aos meus irmãos, Gabrielita, Andrés e Migdalia, vocês são um presente para minha alma, e a força para continuar lutando todos os dias.

À minha amiga Eliana Paola Marin Castaño, por voltar na hora certa!

À Pontifícia Universidade Católica do Rio de Janeiro (PUC), e ao Conselho Nacional de Desenvolvimento Científico e Tecnológico ( $\mathrm{CNPq}$ ), pelo apoio financeiro, que tornou viável a execução desta pesquisa.

E meu mais sincero agradecimento a todos os brasileiros honestos que pagam seus impostos. Concluo mais esta etapa graças a vocês! 


\section{Resumo}

Ramos, Maria Isabel Navarro; Brocchi, Eduardo de Albuquerque; Correia, Rogério Navarro. Síntese e caracterização de ligas Cobre-Níquel contendo nanoparticulas de Alumina. Rio de Janeiro, 2013. 122p. Dissertação de Mestrado - Departamento de Engenharia de Materiais, Pontifícia Universidade Católica do Rio de Janeiro.

Os materiais nanoestruturados têm sido estudados ao longo das últimas décadas, por apresentarem propriedades particulares, promissoras propriedades térmicas, mecânicas e catalíticas, que muitas vezes não estão presentes no material não manométrico. Alguns avanços recentes têm mostrado que estas propriedades podem ser reforçadas pela inclusão de materiais com propriedades diferentes na sua estrutura, formando assim nanocompósitos. Por exemplo, as ligas de $\mathrm{CuNi}$ são muito dúcteis, mas a presença de nanopartículas de $\mathrm{Al}_{2} \mathrm{O}_{3}$ depositadas na matriz pode melhorar consideravelmente a dureza do material. Tal nanocompósito pode ser obtido, por exemplo, através de decomposição térmica nitratos, seguido por redução seletiva com hidrogênio. Nesse contexto, o presente trabalho tem como foco a síntese de ligas de $\mathrm{CuNi}$ e $\mathrm{CuNi}$ com adição de nanoparticulas de $\mathrm{Al}_{2} \mathrm{O}_{3}$, baseados na redução seletiva de $\mathrm{CuO}$ e de $\mathrm{NiO}$ com $\mathrm{H}_{2}$, e óxidos coformados com o óxido de alumínio por meio da decomposição térmica de seus nitratos metálicos. Cálculos termodinâmicos mostraram que a redução de $\mathrm{Cu}$ e Ni pode ser realizada a temperaturas relativamente baixas (400 $\pm 5^{\circ} \mathrm{C}$ ), e também que o processo se desenvolve seletivamente (apenas os óxidos de $\mathrm{Ni}$ e de $\mathrm{Cu}$ reagem nas condições impostas), resultando em compósitos de $\mathrm{CuNi} / \mathrm{Al}_{2} \mathrm{O}_{3}$, que consiste na formação de uma liga $\mathrm{CuNi}$ contendo $1 \%$ de $\mathrm{Al}_{2} \mathrm{O}_{3}$ como finas nanopartículas distribuídas homogeneamente. Precursores e amostras reduzidas foram caracterizados por difração de raios x (DRX) para determinar a natureza das fases individuais presentes (óxidos e ligas), microscopia electrónica de varredura (MEV) como uma primeira aproximação da morfologia das partículas e microscopia eletrônica de transmissão (MET). Os resultados obtidos indicam que a via química proposta resultou satisfatória para a elaboração das ligas $\mathrm{CuNi}$ contendo nanopartículas de $\mathrm{Al}_{2} \mathrm{O}_{3}$ homogeneamente distribuídas. Os resultados obtidos também indicam que, para as condições experimentais impostas tanto a decomposição dos nitratos como as reações de redução alcançaram conversões de $100 \%$.

\section{Palavras-chave}

$\mathrm{CuNi} / \mathrm{Al}_{2} \mathrm{O}_{3}$ compósitos; decomposição térmica de nitratos; redução seletiva com $\mathrm{H}_{2}$. 


\section{Abstrac}

Ramos, Maria Isabel Navarro; Brocchi, Eduardo de Albuquerque (Advisor); Navarro, Rogério Correia (Co-advidor). Synthesis and Characterization of Copper-Niquel alloys containing Alumina nanoparticles. Rio de Janeiro, 2013. 122p. MSc. DissertationDepartamento de Engenharia de Materiais, Pontifícia Universidade Católica do Rio de Janeiro.

Materials containing nanostructured particles have been studied over the last decades in order to take advantage of their promising thermal, mechanical and catalytic properties. Some recent progress has shown that these properties can be further enhanced by the inclusion of materials with different properties in their structure, thereby forming nanocomposites. For instance, $\mathrm{Ni}-\mathrm{Cu}$ alloys are highly ductile, but the presence of $\mathrm{Al}_{2} \mathrm{O}_{3}$ nanoparticles deposited inside the alloy matrix can considerably improve the material's hardness. Such a nanocomposite can be obtained, for example, through nitrate solutions thermal decomposition followed by selective reduction with hydrogen. In this context, the present work focuses on the synthesis of $\mathrm{CuNi}$ alloys and $\mathrm{CuNi} / \mathrm{Al}_{2} \mathrm{O}_{3}$ composites based on the selective reduction of copper and nickel oxides with pure $\mathrm{H}_{2}$, co-formed with aluminum oxide through thermal decomposition of aqueous solutions of their metal nitrates. Thermodynamic computations showed that the $\mathrm{Cu}$ and $\mathrm{Ni}$ reduction can be accomplished at relatively low temperatures $\left(400 \pm 5^{\circ} \mathrm{C}\right)$, and also that the process develops selectively (only the oxides of $\mathrm{Ni}$ and $\mathrm{Cu}$ react at the imposed conditions), resulting in $\mathrm{Cu}-\mathrm{Ni}-\mathrm{Al}_{2} \mathrm{O}_{3}$ composites, consisting in a $\mathrm{Cu}-\mathrm{Ni}$ alloy crystals containing $1 \%$ of $\mathrm{Al}_{2} \mathrm{O}_{3}$ as fine homogeneously distributed nanoparticles. Both the original (co-formed) and reduced oxide samples were characterized using $x$ ray diffraction (XRD) for determining the nature of the individual phases present (oxides and alloys) and scanning electron microscopy (SEM) as a first approach to the investigation of the morphology of the particles. The results indicate that the proposed chemical route resulted in composite materials containing $\mathrm{CuNi}$ alloy and $\mathrm{Al}_{2} \mathrm{O}_{3}$ particles of controllable composition and homogeneously distributed among the samples. The achieved results also suggest that for the imposed experimental conditions both the nitrate decomposition as well as the reduction reactions could be conducted to $100 \%$ conversion.

\section{Keywords}

$\mathrm{CuNi}$ alloys; $\mathrm{CuNi} / \mathrm{Al}_{2} \mathrm{O}_{3}$ composites; nitrate thermal decomposition; $\mathrm{H}_{2}$ selective reduction. 


\section{Sumário}

1 Introdução

1.1. Nanociência, nanotecnologia, nanomateriais e novos materiais.

1.2. Ligas metálicas Cobre -Níquel

1.2.1. Síntese de Nanomateriais

1.2.2. Sínteses de ligas CuNi nanoestruturadas.

1.3. Redução seletiva com hidrogênio.

1.4. Objetivos

2 Considerações teóricas

30

2.1. Aspetos termodinâmicos da decomposição térmica dos nitratos

30

2.1.1. Avaliação termodinâmica relacionada à decomposição térmica dos nitratos de $\mathrm{Cu}, \mathrm{Ni}$ e $\mathrm{Al}[28]$

2.2. Aspetos termodinâmicos da redução dos óxidos de $\mathrm{Cu}, \mathrm{Ni}$ e $\mathrm{Al}$

3.1. Síntese de óxidos isolados (NiO, $\mathrm{CuO}, \mathrm{Al} 2 \mathrm{O} 3$ ) e coformados 
hidrogênio

4.4. Sinterização dos compósitos 84

4.5. Medidas de Dureza 88

5 Caracterização por microscopia eletrônica de transmissão 90

5.1. Princípios de Microscopia eletrônica de transmissão( MET) ncípios de MET [37]

5.3. Microanalise no MET- Espectroscopia de raios $X$ por dispersão de energia (EDXS)

5.4. Resultados

5.4.1. Etapa da dissociação: Óxidos de $\mathrm{Cu}$, $\mathrm{Ni}$ e $\mathrm{Al} 2 \mathrm{O} 3$ coformados 93

5.4.2. Etapa da redução: produtos co-reduzidos A1, B1 e C1

5.5. Influência do tipo de gás na redução dos óxidos

6 Conclusões

7 Referências Bibliográficas

Apêndice 1

Apêndice 2 


\section{Lista de figuras}

Figura 1 - Tecnologias top down e bottom up

Figura 2 - $\triangle G^{\circ}$ em função da temperatura para as reações de dissociação dos nitratos de cobre $\left(\mathrm{Cu}\left(\mathrm{NO}_{3}\right)_{2}\right)$, níquel $\left(\mathrm{Ni}\left(\mathrm{NO}_{3}\right)_{2}\right)$ e de alumínio $\left(\mathrm{Al}\left(\mathrm{NO}_{3}\right)_{3}\right)$.

Figura 3 - Diagrama de especiação para os nitratos de cobre $\left(\mathrm{Cu}\left(\mathrm{NO}_{3}\right)_{2}\right)$, de níquel $\left(\mathrm{Ni}\left(\mathrm{NO}_{3}\right)_{2}\right)$ e de alumínio $\left(\mathrm{Al}\left(\mathrm{NO}_{3}\right)_{3}\right)$ respectivamente

Figura 4 - Variação de $\mathrm{K}_{\text {eq }}$ com a temperatura para a dissociação dos nitratos de níquel, cobre, alumínio

Figura 5 - Diagrama de Elligham ${ }^{[29]}$

Figura 6 - $\Delta G^{\circ}$ em função da temperatura para as reações de redução $\mathrm{CuO}, \mathrm{NiO}$ e $\mathrm{Al}_{2} \mathrm{O}_{3}$

Figura 7 - \% de Hidrogênio vs $\mathrm{T}$ para $\mathrm{CuO}, \mathrm{NiO}$ e $\mathrm{Al}_{2} \mathrm{O}_{3}$

Figura 8 - Diagrama de predominância considerando as condições de equilíbrio das fases

Figura 9 - Diagrama de distribuição das espécies no equilíbrio

Figura 10 - Diagrama de fases do sistema Cu-Ni ${ }^{[29]}$

Figura 11 - Manta aquecedora para dissociação dos nitratos

Figura 12 - Esquema da dissociação de nitratos em óxidos

Figura 13 - Esquema da linha experimental utilizada para as reduções com $\mathrm{H}_{2}$, onde 1 é o variac, 2 é o sistema de saída dos gases, 3 os rotâmetros, 4 os controladores de temperatura, 5 o forno e 6 as barquetes

Figura 14 - Fluxograma da síntese dos nanocompósitos $\mathrm{CuNi} / \mathrm{Al}_{2} \mathrm{O}_{3}$

Figura 15 - Difratograma de amostra obtida após a decomposição do 
$\mathrm{Ni}\left(\mathrm{NO}_{3}\right)_{2}$ a $300^{\circ} \mathrm{C}$

Figura 16 - Imagem via MEV do material obtido via decomposição térmica do $\mathrm{Ni}\left(\mathrm{NO}_{3}\right)_{2}$ a $300^{\circ} \mathrm{C}$

Figura 17 - Media de EDS do produto obtido via decomposição térmica do $\mathrm{Ni}\left(\mathrm{NO}_{3}\right)_{2}$ a $300^{\circ} \mathrm{C}$

Figura 18 - Difratograma de amostra obtida após a decomposição do $\mathrm{Cu}(\mathrm{NO} 3) 2$ a $320^{\circ} \mathrm{C}$

Figura 19 - Imagem MEV do material obtido via decomposição térmica do $\mathrm{Cu}\left(\mathrm{NO}_{3}\right)_{2}$ a $320^{\circ} \mathrm{C}$, com aumento de $2000 \mathrm{X}$ e $1800 \mathrm{X}$ respectivamente

Figura 20 - Media de EDS do produto obtido via decomposição térmica do $\mathrm{Cu}(\mathrm{NO} 3)_{2}$ a $320^{\circ} \mathrm{C}$

Figura 21 - Difratograma de amostra obtida após a decomposição do $\mathrm{Al}(\mathrm{NO} 3) 3.9 \mathrm{H} 2 \mathrm{Os}$ a $500^{\circ} \mathrm{C}$

Figura 22 - Imagem MEV do material obtido via decomposição térmica do $\mathrm{Al}(\mathrm{NO3}) 3.9 \mathrm{H} 2 \mathrm{Os}$ a $500^{\circ} \mathrm{C}$, com aumento de $1500 \mathrm{X}$ e $2500 \mathrm{X}$ respectivamente

Figura 23 - Media de EDS do produto obtido via decomposição térmica do $\mathrm{Al}(\mathrm{NO}) 3.9 \mathrm{H} 2 \mathrm{Os}$ a $500^{\circ} \mathrm{C}$

Figura 24 - Difratograma de amostra A1 obtida após a decomposição térmica dos nitratos de $\mathrm{Cu}, \mathrm{Ni}$ e $\mathrm{Al}$ a $500^{\circ} \mathrm{C}$

Figura 25 - Imagem em MEV da amostra A1 após decomposição térmica dos nitratos de $\mathrm{Cu}, \mathrm{Ni}$ e $\mathrm{Al}$ a $500^{\circ} \mathrm{C}$, com aumentos de $1000 \mathrm{X}$ e $1800 X$ respectivamente

Figura 26 - Media de EDS correspondente a 8 pontos, do produto obtido via decomposição térmica a $500^{\circ} \mathrm{C}$

Figura 27 - Difratograma de amostra B1 obtida após a decomposição 
térmica dos nitratos de $\mathrm{Cu}, \mathrm{Ni}$ e $\mathrm{Al}$ a $500^{\circ} \mathrm{C}$

Figura 28 - Imagem em MEV da amostra B1 após a decomposição térmica dos nitratos de $\mathrm{Cu}, \mathrm{Ni}$ e $\mathrm{Al}$ a $500^{\circ} \mathrm{C}$, com aumentos de a) $1500 \mathrm{X}$ e b) $4000 X$ respectivamente.

Figura 29 - Difratograma de amostra C1 obtida após a decomposição térmica dos nitratos de $\mathrm{Cu}, \mathrm{Ni}$ e $\mathrm{Al}$ a $500^{\circ} \mathrm{C}$

Figura 30 - Imagem em MEV da amostra C1 após a decomposição térmica dos nitratos de $\mathrm{Cu}$, Ni e $\mathrm{Al} \mathrm{a} 500^{\circ} \mathrm{C}$, com aumentos de $800 \mathrm{X}$ e 1200X respectivamente

Figura 31 - Média de EDS correspondente a 9 pontos do produto obtido por decomposição térmica a $500^{\circ} \mathrm{C}$

Figura 32 - Conversão x tempo (min) da redução de $\mathrm{NiO}$ por $\mathrm{H}_{2}$

Figura 33 - Efeito da temperatura sobre $(1-(1-\alpha) 1 / 3)$ com respeito ao tempo segundo modelo topoquímico

Figura 34 - Efeito da temperatura sobre $\operatorname{Ln}(\alpha /(1-\alpha)$ com respeito ao tempo segundo modelo autocatalítico

Figura 35- Ln k vs 1/T segundo modelo topoquímico

Figura 36 - Ln k vs 1/T segundo modelo autocatalitico

Figura 37 - DRX após a redução do NiO 70

Figura 38 - Conversão x tempo (mim) da redução de $\mathrm{CuO}$ por $\mathrm{H}_{2}$

Figura 39 - Efeito da temperatura sobre $(1-(1-\alpha) 1 / 3)$ com respeito ao tempo segundo modelo topoquímico

Figura 40 - Efeito da temperatura sobre $\operatorname{Ln}(\alpha /(1-\alpha)$ com respeito ao tempo segundo modelo autocatalítico

Figura 41 - Ln k vs 1/T segundo modelo topoquímico

Figura 42 - Ln k vs 1/T segundo modelo autocatalitico 
Figura 43 - DRX após a redução do CuO

Figura 44 - Conversão x tempo (mim) da redução de A1 por H2.

Figura 45 - Efeito da temperatura sobre $(1-(1-\alpha) 1 / 3)$ com respeito ao tempo segundo modelo topoquímico

Figura 46 - Efeito da temperatura sobre $\operatorname{Ln}(\alpha /(1-\alpha)$ com respeito ao tempo segundo modelo autocatalitico 76

Figura 47 - Ln k vs 1/T segundo modelo topoquímico 77

Figura 48 - Ln k vs 1/T segundo modelo autocatalitico 78

Figura 49 - Imagem em MEV da amostra A1 após a redução com aumento de $1200 \mathrm{X}$ 78

Figura 50 - DRX após a redução do óxido A1

Figura 51 - Imagem em MEV da amostra A1 após a redução com aumento de $1200 \mathrm{X}$

Figura 52 - Difratograma da amostra B1 após redução

Figura 53 - Imagem em MEV da amostra B1 reduzida com aumento de 1800X.

Figura 54 - Media de EDS obtida de 5 regiões para amostra reduzida $\mathrm{B} 1$

Figura 55 - Difratograma da amostra C1 após redução

Figura 56 - Imagem em MEV da amostra C1 reduzida

82

Figura 57 - Media de EDS para amostra C1 reduzida

Figura 58 - Imagens de MEV das amostras sinterizadas a $1000^{\circ} \mathrm{C}$

por 5 horas a)Amostra A1 b) B1 c) C1

Figura 599 - DRX das amostras A1,B1 e C1 respectivamente, sinterizadas a $1000 \mathrm{C}$ durante 5 horas 
Figura 60 - DRX das amostras $\mathrm{A} 1, \mathrm{~B} 1$ e $\mathrm{C} 1$ num mesmo difratograma

Figura 61 - Correlação entre parâmetro de rede e porcentagem de níquel nas amostras

Figura 62 - Dureza para as amostras com e sem alumina

Figura 63 - Esquema dos feixes coletados para formação da imagem

(a) campo claro, (b) campo escuro e (c) campo escuro centrado

Figura 64 - Padrões de difração típicos: a) Material amorfo, b)

monocristalino e c)policristalino. ${ }^{[37]}$

Figura 65 - Imagens de MET do óxido B1 em campo claro (a) e campo escuro (b).

Figura 66 - Imagem ampliada da Figura 65 (b)

Figura 67 - Mapeamento elementar por dispersão de raios X para o óxido coformado B1.

Figura 68 - Imagens em MET no modo varredura (STEM) do óxido C1, em campo claro (a) - (c), e campo escuro anular (b)- (d).

Figura 69 - Mapeamento elementar por dispersão de raios X para o óxido coformado $\mathrm{C} 1$

Figura 70 - Mapeamento elementar por dispersão de raios $\mathrm{X}$ para o óxido coformado $\mathrm{C} 1$

Figura 71 - Imagens em MET da amostra reduzida A1:Cu49.5 Ni49.5/1AI2O3, a) Imagem STEM campo claro. b) Ampliação da imagem (a), e em destaque (c) as maclas.

Figura 72 - Mapeamento elementar por dispersão de raios $\mathrm{X}$ para a amostra reduzida $\mathrm{A} 1$

Figura 73 - Imagens em STEM -campo claro da amostra reduzida A1 com diferentes aumentos. 
Figura 74 - Imagem de STEM da amostra reduzida A1.

Figura 75 - Amostra B1: Cu4.5Ni4.5-1Al2O3 (4.50-4.50-1\%) a) campo claro b) campo escuro

Figura 76 - Imagem STEM amostra B1 105

Figura 77 - Imagem ampliada da figura 75

Figura 78 - Imagem campo claro/campo escuro da região A a) campo claro b) campo escuro c) ampliação b e d) padrão de difração. O spot escolhido para as análises em campo escuro está indicado no padrão de difração

Figura 79 - Imagem de STEM/campo claro da região C da 75

Figura 80 - Mapeamento elementar por dispersão de raios X para a amostra reduzida B1

Figura 81 - Mapeamento elementar por dispersão de raios $\mathrm{X}$ para a amostra reduzida $\mathrm{C} 1$

Figura 82 - Imagem em MET da amostra A1 a) resfriada com argônio, b) resfriada com hidrogênio. 


\section{Lista de tabelas}

Tabela 1 - Proporções de reagentes necessários para a obtenção dos óxidos e dos produtos finais

Tabela 2 - Caracterização dos óxidos coformados A1, B1, C1 via analise química

Tabela 3 - Variação da massa de oxido com o incremento do fluxo em condições inertes

Tabela 4 - Valores de coeficiente de correlação lineal $\left(R^{2}\right)$, resultantes da linearização do modelo autocatalítico e topoquímico

Tabela 5 - Valores de coeficiente de correlação lineal $\left(R^{2}\right)$, resultantes da linearização do modelo autocatalitico e topoquimico

Tabela 6 - Valores de coeficiente de correlação lineal $\left(R^{2}\right)$, resultantes da linearização do modelo autocatalítico e topoquímico para o óxido $\mathrm{A} 1$

Tabela 7 - Parâmetro de rede e tamanho médio de cristalito para amostras sinterizadas A1, B1, C1 